\title{
High-mobility pentacene OTFT with TaLaO gate dielectric passivated by fluorine plasma
}

\author{
Chuan Yu Han ${ }^{1}$, Wing Man Tang ${ }^{2}$, Cheung Hoi Leung ${ }^{1}$, Chi Ming Che ${ }^{3}$ and Peter T. Lai ${ }^{*, 1}$ \\ ${ }^{1}$ Department of Electrical and Electronic Engineering, the University of Hong Kong, Pokfulam Road, Hong Kong \\ ${ }^{2}$ Department of Applied Physics, the Hong Kong Polytechnic University, Hong Kong \\ ${ }^{3}$ Department of Chemistry, the University of Hong Kong, Pokfulam Road, Hong Kong
}

Keywords Organic thin-film transistor, TaLaO, fluorine plasma, low-frequency noise

* Corresponding author: e-mail laip@eee.hku.hk, Phone: +852 28592691 , Fax: +852 28592691

Pentacene thin-film transistor with high- $\kappa \mathrm{TaLaO}$ as gate dielectric has been fabricated and shows a carrier mobility of 0.73 $\mathrm{cm}^{2} / \mathrm{V} \cdot \mathrm{s}$, much higher than that based on pure $\mathrm{La}_{2} \mathrm{O}_{3}\left(0.43 \mathrm{~cm}^{2} / \mathrm{V} \cdot \mathrm{s}\right)$ due to the smoother surface of the TaLaO film and thus larger pentacene islands grown on it in the initial stage. Moreover, among various times for fluorine-plasma treatment on the $\mathrm{TaLaO}$ gate dielectric, 100 seconds result in the highest carrier mobility of $1.12 \mathrm{~cm}^{2} / \mathrm{V} \cdot \mathrm{s}$ due to (1) smoothest oxide surface achieved by fluorine passivation of oxide traps, as measured by AFM and supported by smallest sub-threshold swing and lowest low-frequency noise; (2) the largest pentacene grains grown on the smoothest oxide surface, as demonstrated by AFM.

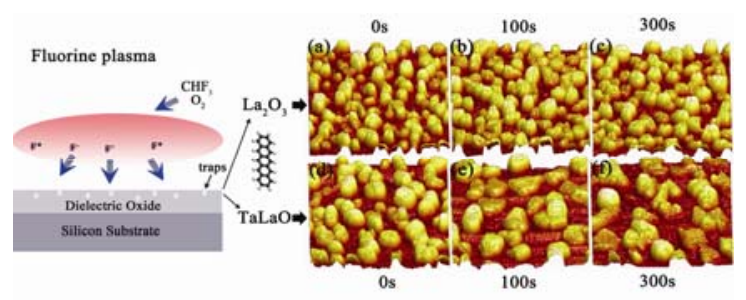

Pentacene islands on on $\mathrm{TaLaO}$ or $\mathrm{La}_{2} \mathrm{O}_{3}$ gate dielectric with different plasma treatment times. 


\section{Introduction}

Organic thin-film transistors (OTFTs) have attracted a wide interest due to their commercial opportunities to be applied in flexible displays, large-area sensors and radio-frequency identification tags[1]. In order to achieve high carrier mobility and low operating voltage for OTFTs, their gate dielectric has to be properly chosen. The operating voltage of OTFTs with $\mathrm{SiO}_{2}$ as gate dielectric is very high, and reducing the oxide thickness to lower the operating voltage inevitably leads to larger gate leakage current. Therefore, high- $\kappa$ material (e.g. HfLaO [2, 3], $\mathrm{HfO}_{2}$ [4], BZT [5] and $\left.\mathrm{Ba}_{0.7} \mathrm{Sr}_{0.3} \mathrm{TiO}_{3}[6]\right)$ as gate dielectric has been proposed as a better alternative for reducing the threshold voltage of the devices.

Both $\mathrm{La}_{2} \mathrm{O}_{3}$ and $\mathrm{Ta}_{2} \mathrm{O}_{5}$ have high dielectric constant and thermal stability [7, 8]. However, $\mathrm{Ta}_{2} \mathrm{O}_{5}$ has small band gap $(4.4 \mathrm{eV})$ while $\mathrm{La}_{2} \mathrm{O}_{3}$ with bigger band gap $(5.5 \mathrm{eV})$ easily absorbs the water vapor in air [9], resulting in rougher surface $[10,11]$. Incorporating other high-lattice-energy oxide is an effective method to suppress the intrinsic moisture absorption of $\mathrm{La}_{2} \mathrm{O}_{3}$ because the moisture absorption is due to the small lattice energy of $\mathrm{La}_{2} \mathrm{O}_{3}[12]$.Hence, in this work, high- $\kappa$ $\mathrm{TaLaO}$ is proposed as the gate dielectric for pentacene OTFT in an attempt to combine the advantages of $\mathrm{La}_{2} \mathrm{O}_{3}$ and $\mathrm{Ta}_{2} \mathrm{O}_{5}$ while suppressing their undesirable properties. Together with appropriate fluorine-plasma treatment time on the $\mathrm{TaLaO}$ gate dielectric, the OTFT can achieve high performance, e.g. high carrier mobility, small sub-threshold swing (SS) and low threshold voltage.

\section{Experimental Section}

Pentacene OTFTs with TaLaO or $\mathrm{La}_{2} \mathrm{O}_{3}$ gate dielectric were fabricated by employing the bottom-gate top-contact configuration. Heavily doped silicon wafers (n-type, $<100>$, resistivity of $0.5 \sim 1.0 \Omega \cdot \mathrm{cm}$ ) were cleaned by the standard RCA method and dipped in dilute $\mathrm{HF}$ acid $(2 \%)$ to remove the native oxide.

Table 1 Parameters of the OTFTs with $\mathrm{TaLaO}$ or $\mathrm{La}_{2} \mathrm{O}_{3}$ gate dielectric treated in fluorine plasma for different times.

\begin{tabular}{lcccccc}
\hline Sample & $\mathrm{A}$ & $\mathrm{B}$ & $\mathrm{C}$ & $\mathrm{D}$ & $\mathrm{E}$ & $\mathrm{F}$ \\
\hline Gate Dielectric & $\mathrm{La}_{2} \mathrm{O}_{3}$ & $\mathrm{La}_{2} \mathrm{O}_{3}$ & $\mathrm{La}_{2} \mathrm{O}_{3}$ & $\mathrm{TaLaO}$ & $\mathrm{TaLaO}$ & $\mathrm{TaLaO}$ \\
Plasma Treatment Time $(\mathrm{s})$ & 0 & 100 & 300 & 0 & 100 & 300 \\
Carrier Mobility $\left(\mathrm{cm}^{2} / \mathrm{V} \cdot \mathrm{s}\right)$ & 0.432 & 0.280 & 0.273 & 0.736 & 1.12 & 0.853 \\
Threshold Voltage $(\mathrm{V})$ & -2.82 & -3.06 & -3.30 & -1.94 & -1.79 & -1.99 \\
$\mathrm{SS}(\mathrm{V} / \mathrm{dec})$ & 0.493 & 0.557 & 0.514 & 0.511 & 0.383 & 0.439 \\
$\mathrm{On} / \mathrm{Off}$ Current Ratio $\left(10^{4}\right)$ & 0.353 & 0.248 & 0.241 & 0.767 & 2.13 & 1.22 \\
$\mathrm{I}_{\mathrm{D}} @ \mathrm{~V}_{\mathrm{DS}}=\mathrm{V}_{\mathrm{GS}}=-5 \mathrm{~V}(\mu \mathrm{A})$ & 1.56 & 0.817 & 0.651 & 4.87 & 7.83 & 5.83 \\
$\mathrm{C}_{\mathrm{ox}}\left(\mu \mathrm{F} / \mathrm{cm}^{2}\right)$ & 0.238 & 0.228 & 0.217 & 0.204 & 0.215 & 0.228 \\
$\mathrm{t}_{\mathrm{ox}}(\mathrm{nm})$ & 32.1 & 31.0 & 31.9 & 40.1 & 40.9 & 40.9 \\
$\kappa \mathrm{Value}$ & 8.62 & 8.00 & 7.81 & 9.26 & 9.95 & 10.3 \\
$\mathrm{Roughness}^{13}$ of Gate Dielectric $(\mathrm{nm})$ & 0.817 & 1.38 & 1.50 & 0.327 & 0.255 & 0.268 \\
$\mathrm{~N}_{\mathrm{SS}}\left(10^{13} / \mathrm{cm}^{2}\right)$ & 1.08 & 1.19 & 1.03 & 0.966 & 0.794 & 0.908 \\
$\mathrm{~S}_{\mathrm{ID}} @ 1 \mathrm{~Hz}\left(10^{-19} \mathrm{~A}^{2} / \mathrm{Hz}\right)$ & 1.55 & 8.35 & 0.673 & 6.25 & 0.793 & 1.30 \\
$\mathrm{~g}_{\mathrm{m}}(\mu \mathrm{S})$ & 0.940 & 1.01 & 0.817 & 1.97 & 8.10 & 1.42 \\
$\mathrm{~N}_{\mathrm{T}}\left(10^{19} / \mathrm{cm}^{3}\right)$ & 3.78 & 16.9 & 1.98 & 2.98 & 0.0235 & 1.33 \\
Surface Energy $(\mathrm{mN} / \mathrm{m})$ & 36.2 & 68.6 & 69.7 & 35.1 & 69.7 & 71.3 \\
Dispersion Component $(\mathrm{mN} / \mathrm{m})$ & 35.5 & 37.9 & 35.3 & 33.9 & 35.0 & 36.2 \\
Polar Component $(\mathrm{mN} / \mathrm{m})$ & 0.627 & 30.7 & 34.4 & 1.15 & 34.7 & 35.1 \\
Average Area of Pentacene Islands $\left(\mu \mathrm{m}^{2}\right)$ & 0.061 & 0.082 & 0.086 & 0.093 & 0.168 & 0.142 \\
\hline
\end{tabular}

Next $\mathrm{La}_{2} \mathrm{O}_{3}$ (for samples A to $\mathrm{C}$ ) or TaLaO (for samples $\mathrm{D}$ to $\mathrm{F}$ ) was sputtered at room temperature by a radio-frequency sputterer (Denton Vacuum LLC Discovery 635). Then, the wafers were annealed in $\mathrm{N}_{2}$ at $400{ }^{\circ} \mathrm{C}$ and a flow rate of 1000 $\mathrm{mL} / \mathrm{min}$ for $20 \mathrm{~min}$. After that, the wafers were treated by a fluorine plasma with $\mathrm{CHF}_{3}$ at $10 \mathrm{sccm}$ and $\mathrm{O}_{2}$ at $1 \mathrm{sccm}$ for 4 different times ( $0 \mathrm{~s}$ for samples A and D, $100 \mathrm{~s}$ for samples B and E, $300 \mathrm{~s}$ for samples $\mathrm{C}$ and F). Next, 30-nm pentacene ( $99 \%$ purity, purchased from Sigma-Aldrich without purification) film was evaporated on the dielectrics by an evaporator (Edwards Auto 306$)$ in high vacuum $\left(4 \times 10^{-6}\right.$ torr) at a deposition rate of $1.2 \mathrm{~nm} / \mathrm{min}$, as monitored by a quartz-crystal oscillator. Lastly, drain and source electrodes were formed on the pentacene film by gold evaporation through a shadow mask. The width and length of the channel on the shadow mask were $200 \mu \mathrm{m}$ and $30 \mu \mathrm{m}$, respectively. On the other 
hand, 3-nm (about 2 monolayers) pentacene film evaporated on the dielectrics was used to study its growth mechanism in the initial stage.

The current-voltage (I-V) characteristics and low-frequency noise (LFN) of the transistors were measured by HP 4145B semiconductor parameter analyzer, Berkeley Technology Associates FET Noise Analyzer Model 9603 and HP 35665A Dynamic Signal Analyzer. Capacitance-voltage measurements were performed on MOS capacitors at a frequency of $1 \mathrm{MHz}$ with HP 4284A LCR meter. The thickness of the dielectric films $\left(\mathrm{t}_{\mathrm{ox}}\right)$ was measured by a Wvase 32 ellipsometer. A Nanopics 2100 atomic force profilometer and Veeco multi-mode scanning probe microscope were employed to record the surface morphology of the dielectrics and 3-nm pentacene films, respectively.

The surface energy of the samples was evaluated using a KRUSS DAS 30 goniometer by the method proposed by Owens and Wendt [13] based on measuring the contact angle of 3 liquids (water, diiodomethane and glycerol) at $20^{\circ} \mathrm{C}$ in static mode.

\section{RESULTS AND DISCUSSION}

Fig. 1 shows the transfer characteristics of the pentacene OTFTs with TaLaO or $\mathrm{La}_{2} \mathrm{O}_{3}$ gate dielectric. The carrier mobility is calculated from the slope of the plot of $\left(I_{D}\right)^{1 / 2}$ versus $V_{G}$ in the saturation regime. The sub-threshold swing is defined as

$S S=\frac{d V_{G}}{d\left(\log I_{D}\right)}$

Assuming that both the density of deep bulk trap and density of (deep) interface trap are independent of energy, the sub-threshold swing can be written as [14]

$S S=\frac{k T \ln 10}{q}\left[1+\frac{q}{C_{o x}} N_{S S}\right]$

where $N_{S S}$ is the density of deep bulk trap and interface trap per unit area and unit energy, $k$ the Boltzmann's constant, $q$ the electron charge, $C_{o x}$ the oxide capacitance per unit area and $T$ the temperature in Kelvin. Therefore, the trap density can be extracted from $S S$ by using equation (2).

The key parameters of the six devices are listed in Table 1. For the OTFTs with TaLaO gate dielectric, the carrier mobility first increases with the plasma treatment times and then decreases after a plasma treatment for $100 \mathrm{~s}$. In contrast, the mobility of OTFTs with $\mathrm{La}_{2} \mathrm{O}_{3}$ gate dielectric decreases with the plasma treatment times. The carrier mobility has a strong relation with the surface roughness of gate dielectric listed in Table 1 and the size of pentacene

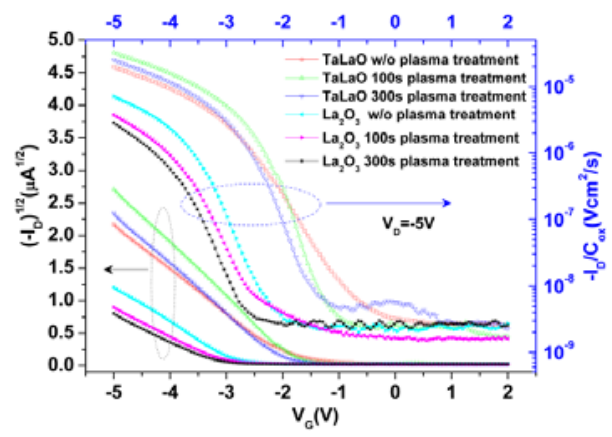

Figure 1. Transfer characteristics of the OTFTs with $\mathrm{TaLaO}$ or $\mathrm{La}_{2} \mathrm{O}_{3}$ gate dielectric treated in fluorine plasma for different times.

islands in their initial growth stage shown in Fig. 2. The TaLaO film with 100-s plasma treatment obtains the smoothest surface among all the samples by fluorine passivation of oxide traps, resulting in the largest pentacene islands in the initial growth stage and corresponding the highest carrier mobility due to least grain-boundary charge trapping and scattering [15], and least surface scattering caused by rough dielectric surface [16]. However, too long plasma-treatment time can cause ion-induced damage to the dielectric surface and thus decrease the carrier mobility. On the other hand, with increasing plasma treatment time, the surface of the $\mathrm{La}_{2} \mathrm{O}_{3}$ film gets rougher and thereby the carrier mobility of the OTFTs decreases, although the sizes of pentacene islands on the plasma-treated $\mathrm{La}_{2} \mathrm{O}_{3}$ are nearly the same. The OTFTs with $\mathrm{TaLaO}$ gate dielectric have higher carrier mobility than their counterparts with $\mathrm{La}_{2} \mathrm{O}_{3}$ gate dielectric. This is because (1) $\mathrm{La}_{2} \mathrm{O}_{3}$ can easily absorb the water vapor in the air and thus its surface becomes rougher [11] as listed in Table 1, resulting 
in stronger surface-roughness scattering, and Ta incorporated in $\mathrm{La}_{2} \mathrm{O}_{3}$ can stabilize the ternary oxide due to the higher lattice energy of $\mathrm{Ta}_{2} \mathrm{O}_{5}$, thus resulting in smoother surface compared with $\mathrm{La}_{2} \mathrm{O}_{3} ;(2)$ rougher surface has more nucleation sites for the growth of pentacene grains, thus enhancing the grain-boundary charge trapping and scattering. On the contrary, the smoother surface of $\mathrm{TaLaO}$ results in the growth of larger pentacene islands, leading to higher carrier mobility. Moreover, the $\mathrm{TaLaO}$ gate dielectric has higher durability against plasma-induced lattice damage. This is possibly because (1) Ta-O bond energy $(530.3 \mathrm{eV})$ is higher than the bond energy of $\mathrm{La}_{2} \mathrm{O}_{3}(529.1 \mathrm{eV})$; (2) $\mathrm{La}_{2} \mathrm{O}_{3}$ absorbs the moisture in air, thus degrading the quality of the $\mathrm{La}_{2} \mathrm{O}_{3}$ dielectric [10]. The threshold voltage of the OTFTs with TaLaO is about $-2 \mathrm{~V}$, which is smaller than that of the OTFTs with $\mathrm{La}_{2} \mathrm{O}_{3}$ due to the higher $\kappa$ value of TaLaO gate dielectric (see Table 1).

Pentacene film growth on the dielectric in the initial stage is crucial to the carrier mobility of organic device because charge transport in the device is mainly confined to the first few monolayers of the organic film in close proximity to the gate dielectric [17]. In order to study the difference

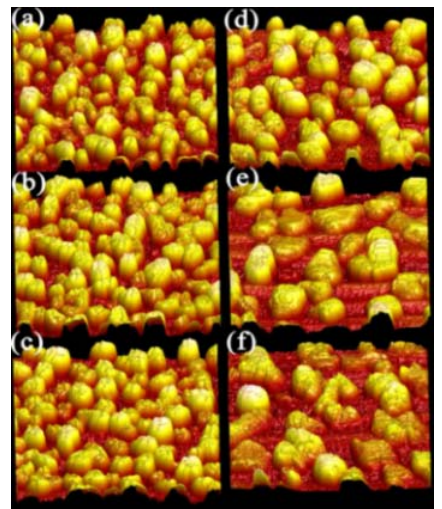

Figure 2. AFM images $(3 \mu \mathrm{m} \times 3 \mu \mathrm{m})$ of 3 -nm pentacene film grown on $\mathrm{TaLaO}$ (right column) or $\mathrm{La}_{2} \mathrm{O}_{3}$ (left column) gate dielectric with different plasma treatment times: (a) and (d) for $0 \mathrm{~s}$, (b) and (e) for $100 \mathrm{~s}$, (c) and (f) for $300 \mathrm{~s}$.

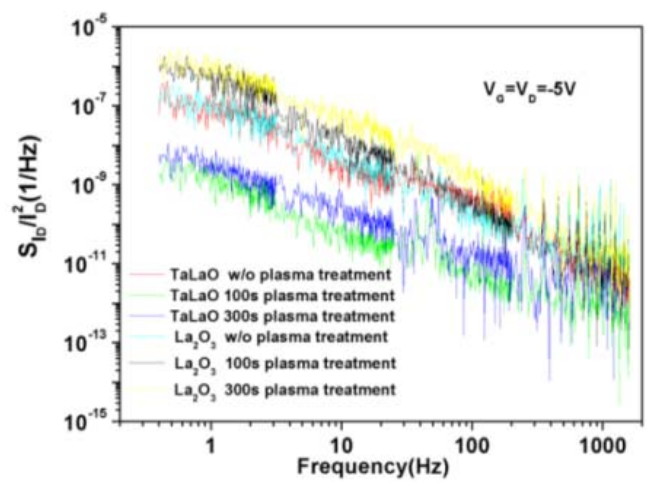

Figure 3. Low-frequency noise characteristics of the OTFTs with $\mathrm{TaLaO}$ or $\mathrm{La}_{2} \mathrm{O}_{3}$ gate dielectric treated in fluorine plasma for different times.

in the initial pentacene growth on the TaLaO and $\mathrm{La}_{2} \mathrm{O}_{3}$ dielectrics, the AFM images of 3-nm (about 2 monolayers) pentacene thin films are shown in Fig. 2. Because of the smoother surface, pentacene islands on TaLaO are much larger than those on $\mathrm{La}_{2} \mathrm{O}_{3}$, resulting few interface traps and high carrier mobility. The plasma treatment can increase the size of the pentacene islands on both $\mathrm{TaLaO}$ and $\mathrm{La}_{2} \mathrm{O}_{3}$ in the initial stage because it can increase the surface energy (see Table 1) by passivating the oxide traps (at the surface and in the bulk of the gate dielectric) with fluorine atoms, thus resulting in less nucleation sites [18]. Moreover, it is clear that the pentacene growth pattern is typically in 3-D clusters, concurring with the Vollmer-Weber growth model $[19,20]$. 
LFN is closely related to the traps located at/near the dielectric/pentacene interface which cause current fluctuation in the conduction channel [21]. The fluctuation in carrier number caused by the trapping and de-trapping processes in the gate dielectric plays a key role in the fluctuation of the drain current. To study the relation between LFN and traps at the interface, the $1 / f$ noise (or flicker noise) model proposed by Ghibaudo et al. [22] is widely accepted and expressed by:

$$
\frac{S_{I_{D}}(f)}{I_{D}^{2}}=\left(1 \pm \alpha \mu_{e f f} C_{o x} \frac{I_{D}}{g_{m}}\right) \frac{g_{m}^{2}}{I_{D}^{2}} \frac{q^{2} k T N_{T}}{W L C_{o x}^{2} f^{\gamma}}
$$

where $S_{I_{D}}(f)$ is the power spectral density of the drain current, $g_{m}$ the transconductance, $\alpha$ the constant correlated with the sensitivity of carrier mobility to interface-charge Coulomb scattering, $\gamma$ the slope of the power spectral density, $f$ the frequency. Therefore, the normalized powerdensity of the drain current, $S_{I_{D}}(f) / I_{D}^{2}$, is proportional to the trap density at the interface. For OTFT based on high- $\kappa$ dielectric, the fluctuation in carrier number caused by the trapping and detrapping of charge carriers in the gate dielectric dominates the fluctuation in the drain current, and thus the cross correlation coefficient $\alpha$ is close to zero [23]. Therefore, as listed in Table 1, the trap density $\left(N_{T}\right)$ can be calculated after the power spectral density of drain current at a frequency of $1 \mathrm{~Hz}$ is measured. Among the samples, the sample E exhibits the lowest LFN as shown in Fig. 3, indicating that with 100-s fluorine plasma passivation, the TaLaO surface has least traps, lending support to the highest carrier mobility of the sample.

Without the plasma treatment, the carrier mobility of the OTFT with TaLaO (sample D) is comparable with that of OTFT with HfLaO[2] and better than that of OTFT with $\mathrm{Ta}_{2} \mathrm{O}_{5}$ [24]. In addition, with the plasma treatment, the carrier mobility of the OTFT with TaLaO (sample E) is among the highest values $(>1.0 \mathrm{~cm} / \mathrm{V} \cdot \mathrm{s}$ ) achieved by high- $\kappa$ gate dielectrics (e.g. $\mathrm{Ba}_{0.7} \mathrm{Sr}_{0.3} \mathrm{TiO}_{3}[6]$, LaAlO[25]) and is comparable to $1.25 \mathrm{~cm}^{2} / \mathrm{V} \cdot \mathrm{s}$ of OTFT with $\mathrm{SAM}$-modified $\mathrm{SiO}_{2}[26]$. This demonstrates that fluorinated $\mathrm{TaLaO}$ is a promising gate dielectric for low-voltage and high-mobility OTFTs.

\section{CONCLUSION}

In conclusion, we have applied $\mathrm{TaLaO}$ or $\mathrm{La}_{2} \mathrm{O}_{3}$ as the gate dielectric of pentacene OTFT and also investigated the effects of treating their surfaces in a fluorine plasma on the performance of these devices. With a 100-s plasma treatment, the OTFT with TaLaO shows the highest carrier mobility and smallest sub-threshold swing. The results suggest that fluorinated $\mathrm{TaLaO}$ is a promising gate dielectric for high-performance pentacene OTFTs.

\section{Acknowledgements}

This work is supported by the URC for Seed Fund for Strategic Research Theme of HKU on New Materials, and the University Development Fund (Nanotechnology Research Institute, 00600009) of the University of Hong Kong. The authors would like to thank Dickey Ma for his help. 


\section{References}

[1] T.W. Kelley, P.F. Baude, C. Gerlach, D.E. Ender, D. Muyres, M.A. Haase, D.E. Vogel, S.D. Theiss, Chem Mater 16, 4413-4422 (2004).

[2] M.F. Chang, P.T. Lee, S.P. McAlister, A. Chin, IEEE Electr Device L 29, 215-217 (2008).

[3] M.F. Chang, P.T. Lee, S.P. McAlister, A.S. Chin, IEEE Electr Device L 30, 133-135 (2009).

[4] O. Acton, G.G. Ting, P.J. Shamberger, F.S. Ohuchi, H. Ma, A.K. Jen, ACS Appl Mater Interfaces 2, 511-520 (2010).

[5] C.D. Dimitrakopoulos, S. Purushothaman, J. Kymissis, A. Callegari, J.M. Shaw, Science 283, 822-824 (1999).

[6] Z.R. Wang, J.Z. Xin, X.C. Ren, X.L. Wang, C.W. Leung, S.Q. Shi, A. Ruotolo, P.K.L. Chan, Org Electron 13, 1223-1228 (2012).

[7] G. He, Z.Q. Sun, G. Li, L.D. Zhang, Crit Rev Solid State 37, 131-157 (2012).

[8] G.D. Wilk, R.M. Wallace, J.M. Anthony, J Appl Phys 89, 5243-5275 (2001).

[9] J. Robertson, Rep Prog Phys 69, 327-396 (2006).

[10] Y. Zhao, Materials 5, 1413-1438 (2012).

[11] Y. Zhao, M. Toyama, K. Kita, K. Kyuno, A. Toriumi, Appl Phys Lett 88, 072904 (2006).

[12] Y. Yokogawa, M. Yoshimura, S. Somiya, J Mater Sci Lett 10, 509-511 (1991).

[13] D.K. Owens, R.C. Wendt, J Appl Polym Sci 13, 1741-1747 (1969).

[14] W.L. Kalb, B. Batlogg, Phys Rev B 81, 035327 (2010).

[15] L. Ke, S. Bin Dolmanan, L. Shen, C. Vijila, S.J. Chua, R.Q. Png, P.J. Chia, L.L. Chua, P.K.H. Ho, J Appl Phys 104,124502 (2008).

[16] D. Knipp, R.A. Street, A. Volkel, J. Ho, J Appl Phys 93, 347-355 (2003).

[17] R. Ruiz, B. Nickel, N. Koch, L.C. Feldman, R.F. Haglund, A. Kahn, G. Scoles, Phys Rev B 67, 125406 (2003).

[18] B.J. Song, K. Hong, W.K. Kim, K. Kim, S. Kim, J.L. Lee, J Phys Chem B 114, 14854-14859 (2010).

[19] S.Y. Yang, K. Shin, C.E. Park, Adv Funct Mater 15, 1806-1814 (2005).

[20] S. Verlaak, S. Steudel, P. Heremans, D. Janssen, M.S. Deleuze, Phys Rev B 68, (2003).

[21] L. Ke, S. Bin Dolmanan, C. Vijila, S.J. Chua, Y.H. Han, T. Mei, Ieee T Electron Dev 57, 385-390 (2010).

[22] G. Ghibaudo, O. Roux, C. Nguyenduc, F. Balestra, J. Brini, Physica Status Solidi a-Applied Research 124, $571-581$ (1991).

[23] Y. Xu, T. Minari, K. Tsukagoshi, J. Chroboczek, F. Balestra, G. Ghibaudo, Solid State Electron 61, 106-110 (2011).

[24] Y.T. Jeong, A. Dodabalapur, Appl Phys Lett 91, 193509 (2007).

[25] S. Yaginuma, J. Yamaguchi, K. Itaka, H. Koinuma, Thin Solid Films 486, 218-221 (2005).

[26] H.S. Seo, Y.S. Jang, Y. Zhang, P. Syed Abthagir, J.H. Choi, Org Electron 9, 432-438 (2008). 